Eur J Clin Chem Clin Biochem

1995; 33:513-517

(c) 1995 Walter de Gruyter \& Co.

Berlin $\cdot$ New York

\title{
Differences Between Enzymatic and Diazo Methods for Measuring Direct Bilirubin
}

\author{
By Katsushi Nakayama
}

Department of Laboratory Medicine, Niigata University School of Medicine, Niigata, Japan

(Received January 31/May 9, 1995)

Summary: An orthodox diazo method is popularly used for measuring bilirubin. On the other hand, an enzymatic method which employs bilirubin oxidase, has also been in use for considerable time. We have often found disparities between direct bilirubin values obtained with the enzymatic and the diazo methods. To determine the cause of these disparities, bilirubin subfractions were analysed and classified into two types by HPLC. Samples showing great differences contained conjugated, unconjugated and $\delta$ bilirubins (type I), while samples showing only small differences contained almost exclusively unconjugated bilirubin and $\delta$ bilirubin (type II). Conjugated bilirubin is therefore largely responsible for the differences observed between the two methods. Particularly marked differences were found for bile (in which all the bilirubin is conjugated) and for synthetic conjugated bilirubin. Bilirubin oxidase decreases the absorbance at $450 \mathrm{~nm}$ when it catalyses oxidation of bilirubin, but after the oxidation of synthetic conjugated bilirubin at $\mathrm{pH} 3.7$ another peak appeared at $450 \mathrm{~nm}$, as shown by HPLC and spectrophotometry, but not when the reaction was performed at $\mathrm{pH} 7.2$, namely under conditions permitting complete oxidation. Incomplete oxidation products of conjugated bilirubin are responsible for the disparity. Care is therefore needed in the clinical interpretation of direct bilirubin values measured by the enzymatic method.

\section{Introduction}

Orthodox diazo methods, such as the Malloy-Evelyn method (1), are popular for the measurement of bilirubin. An enzymatic method $(2,3)$ is also used for measuring total and direct bilirubin, based on the decrease in absorbance at $450 \mathrm{~nm}$ when bilirubin is oxidized to biliverdin in the presence of bilirubin oxidase $\left.(4,5) .{ }^{1}\right)$ But this is not a complete replacement for the diazo method, because large differences in direct bilirubin values are often found between the two methods, while the values for total bilirubin are largely in agreement.

The aim of this study is to clarify the cause of this difference. First hyperdirectbilirubinaemia was divided into two types. Type I showed large differences, while type II showed only small differences. The bilirubin subfractions of these two types were investigated by HPLC.

1) Enzyme: Bilirubin oxidase (EC 1.3.3.5)
In addition, ditaurobilirubin (6), a synthetic conjugated bilirubin, was analysed by the bilirubin oxidase reaction, by HPLC and by spectrophotometry.

In the present study HPLC proved to be a potent tool for analysing bilirubin subfractions and for identifying the sources of absorbance changes in bilirubin oxidation.

\section{Materials and Methods}

An HPLC, LC-6A (Shimadzu Corporation, Kyoto, Japan) was used with a Micronex RP-30 column (Sekisui Chemical Co., Osaka, Japan) for bilirubin subfractionation. The HITACHI 736-60 (HITACHI, Ltd., Tokyo, Japan) was used for spectrophotometric measurements.

Icteric sera (total bilirubin $\geqq 34 \mu \mathrm{mol} / /$, direct bilirubin $\geqq 11$ $\mu \mathrm{mol} / \mathrm{l})$ were collected at random from the laboratory of Niigata University Hospital. The sera were neither haemolysed nor chylous.

Bile was obtained from an otherwise normal gall-stone patient during his operation. It was then filtered through a MILLEX-HV 0.45 $\mu \mathrm{m}$ Filter Unit (Millipore Corporation, Bedford, MA USA). 
Bilirubin.C (ditaurobilirubin) of "Interference Check.A" (INTERNATIONAL REAGENTS CORPORATION, Kobe, Japan) served as conjugated bilirubin.

\section{HPLC subfractionation}

HPLC subfractionation was done as described by Adachi et al. (7). Briefly, a sample was mixed with an equal amount of $0.1 \mathrm{~mol} / \mathrm{l}$ acetic acid reagent, then passed through a MILLEX-HV $0.45 \mu \mathrm{m}$ filter to eliminate any fibrin precipitates. A $20-\mu \mathrm{l}$ aliquot of this filtrate was injected into the HPLC. Finally, the absorbance was measured at $450 \mathrm{~nm}$. Assay variation was $7.7 \%(n=5)$ withinday, $3.5 \%(n=10)$ between-run.

\section{Diazo method}

An alkaline azobilirubin method kit, Nescauto BIL-V3 (Nippon Shoij Kaisha, Ltd., Osaka, Japan) (8), was used for the diazo method. The diazo reagent of this kit contains sulphanilic acid and sodium nitrite dissolved in hydrochloric acid solution, as in the Malloy-Evelyn reagent, but the accelerator consists of urea, sodium acetate and glycine. The absorbance gain was detected at $456 \mathrm{~nm}$ on an autoanalyser HITACHI 736-60. The intra-assay and the interassay precision of the total bilirubin assay were $2.9 \%$ and $3.7 \%$ respectively, whereas those of the direct bilirubin assay were $2.3 \%$ and $2.4 \%$, respectively, for the analysis of $(n=4)$ serum samples.

\section{Enzymatic method}

An enzymatic method kit, Nescauto BIL-VE (Nippon Shoji Kaisha, Ltd., Osaka, Japan) (9) was used, and decreases at $450 \mathrm{~nm}$ were measured on a HITACHI 736-60. The intra-assay and the interassay precision for total bilirubin were $2.7 \%$ and $2.0 \%$, respectively, whereas those for direct bilirubin were $3.7 \%$ and $4.5 \%$, respectively, for the analysis of $(n=4)$ serum samples.

\section{Results}

\section{Bilirubin subfractionation in serum}

Figure la shows four bilirubin subfractions detected on HPLC: $\alpha$ (unconjugated bilirubin), $\beta$ (monoconjugated bilirubin), $\gamma$ (biconjugated bilirubin) and $\delta$ ( $\delta$ bilirubin $(10-12)$, which is a covalently albumin-bound bilirubin). Their retention times (mean \pm SD) were 34.7 $\pm 0.1 \mathrm{~min}, 29.3 \pm 0.1 \mathrm{~min} \& 28.6 \pm 0.1 \mathrm{~min}$ (biphasic), $24.9 \pm 0.1 \mathrm{~min}$, and $20.2 \pm 0.3 \mathrm{~min}$, respectively.

\section{Comparison of bilirubin values}

Figure $2 \mathrm{~b}$ shows the direct bilirubin values of eight serum samples that show large differences between the two methods. The average difference (|direct bilirubin $_{\text {diazo }}-$ direct bilirubin $_{\text {enzymatic }}$ )/direct bilirubi$\mathrm{n}_{\text {enzymatic }}$ was $53 \%$. The average difference for total bilirubin (|total bilirubin diazo - total bilirubin enzymatic $) /$ total bilirubin $_{\text {enzymatic }}$ was $7 \%$ as shown in figure $2 a$. Their chromatograms always show all the four main peaks (fig. 1a) (type I). Conversely, figure 3 shows three serum samples that show only small differences between the two methods (type II); the average differences for direct and total bilirubin were $14 \%$ and $3 \%$, respectively.

\section{Bilirubin subfractionation in bile}

Figure 1c shows a chromatogram of bile. Its chief constituents were $\beta$ (retention time: $28.4 \mathrm{~min} \& 29.6 \mathrm{~min}$ )
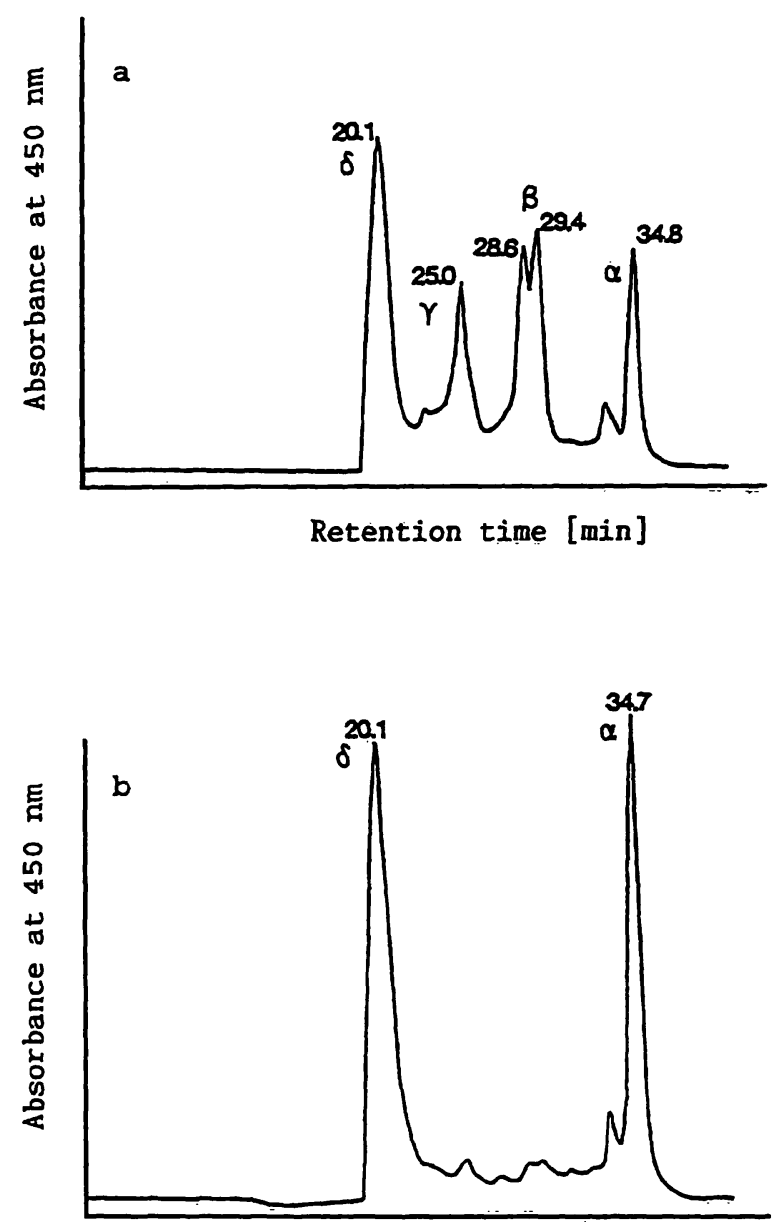

Retention time [min]

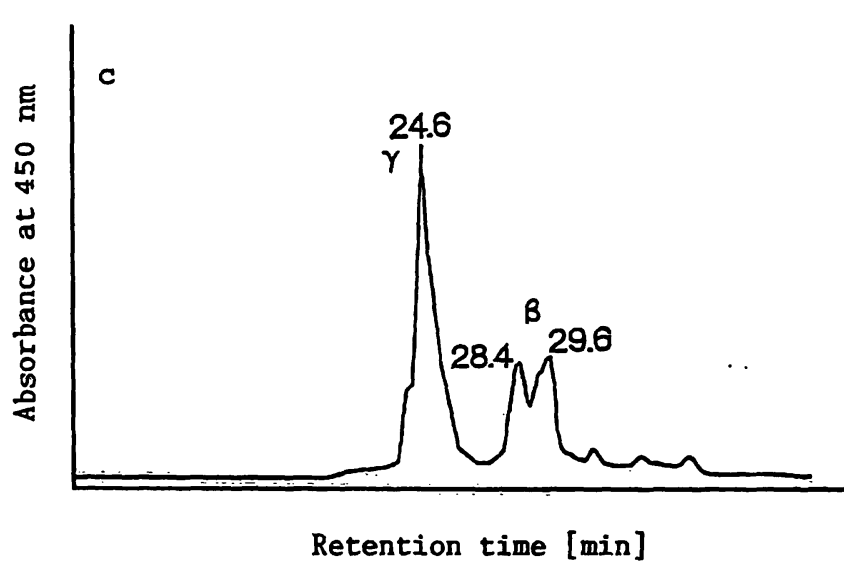

Fig. 1 Chromatogram of icteric sera and bile.

(a) Sera showing large methodical differences. .

(b) Sera showing small differences.

(c) Bile.

The number and Greek letter at each peak indicate the retention time (min) and the subfraction name, respectively. 
and $\gamma$ (retention time: $24.6 \mathrm{~min}$ ). The difference between the two methods was substantial (data not shown).

\section{Absorbance changes of ditaurobilirubin before and after each bilirubin assay}

To investigate the disparity in direct bilirubin values, ditaurobilirubin was used as a conjugated bilirubin. The absorbance due to ditaurobilirubin was detected in different concentrations before and after the reaction (measured on the autoanalyzer HITACHI 736-60 for the diazo and enzymatic reactions at $546 \mathrm{~nm}$ and at $450 \mathrm{~nm}$, respectively). Figure 4 indicates that the baseline of the enzymatic reaction rose according to the concentration of ditaurobilirubin, while that of the diazo method was almost flat.

Changes in chromatographic behaviour due to the bilirubin oxidase reaction

The chromatograms of ditaurobilirubin before and after the addition of bilirubin oxidase at $\mathrm{pH} 3.7$ and $\mathrm{pH} 7.2$ were
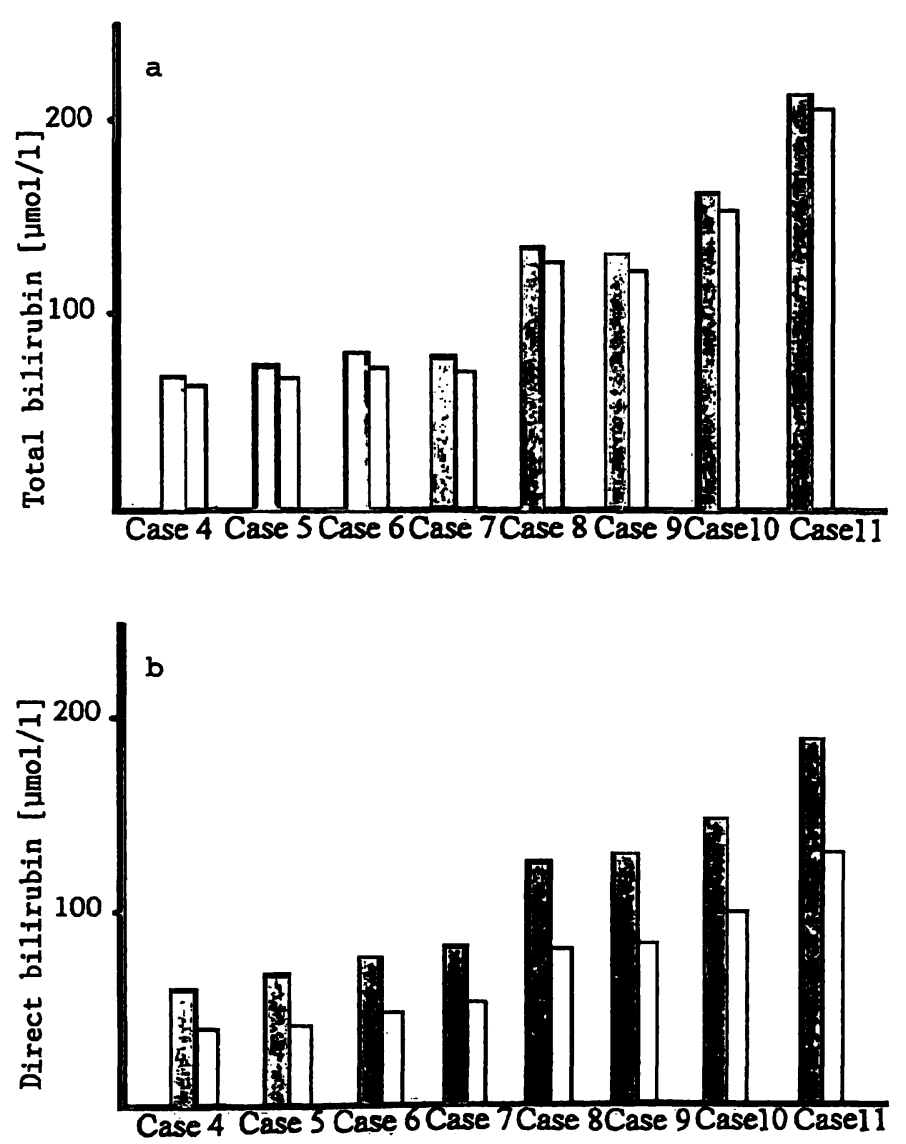

Fig. 2 Comparison of bilirubin values in type I hyperdirectbilirubinaemia.
(a) Total bilirubin values by the diazo method ( $\nabla$ ) and those by the enzymatic method ( $\square$ ).
(b) Direct bilirubin values by the diazo method ( $\square)$ and those by the enzymatic method ( $\square$ ).
Mean difference (defined in the text) in direct bilirubin values was $53 \%$ whereas that in total bilirubin values was $7 \%$.

compared. At pH 3.7 (for direct bilirubin measurement), the $\beta$ and $\gamma$ peaks completely disappeared after addition of bilirubin oxidase (fig. 5), but other material absorbing at $450 \mathrm{~nm}$ appeared during the retention time 2.9 to 3.5
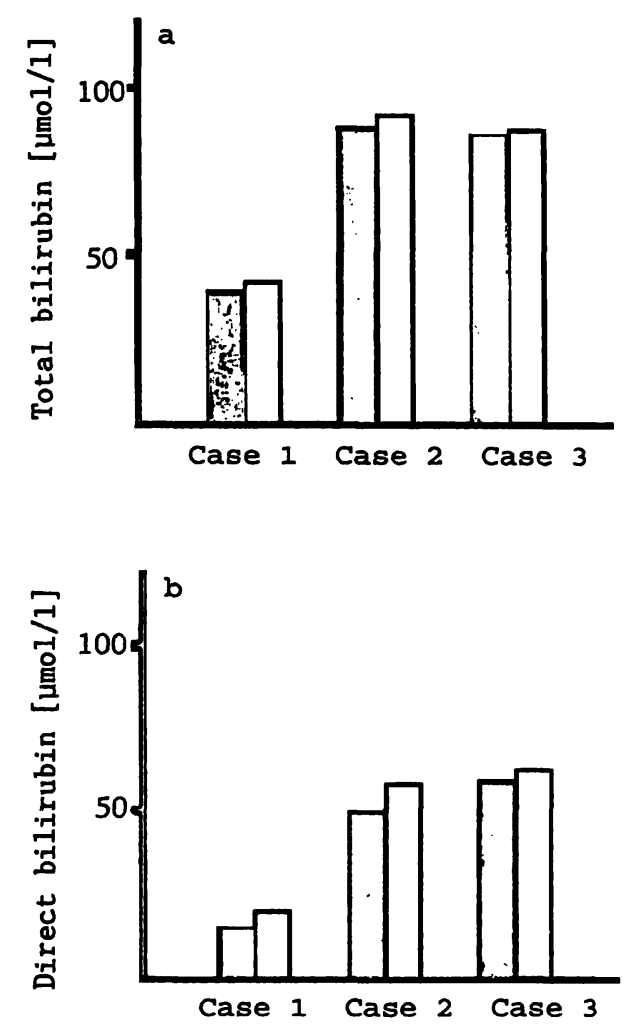

Fig. 3 Comparison of bilirubin values in type II hyperdirectbilirubinaemia.

(a) Total bilirubin values by the diazo method (ם) and those by the enzymatic method ( $\square$ ).

(b) Direct bilirubin values by the diazo method ( $\boldsymbol{\square}$ ) and those by the enzymatic method ( $\square$ ).

Mean difference (defined in the text) in direct bilirubin values was $14 \%$ whereas that in total bilirubin values was $3 \%$.

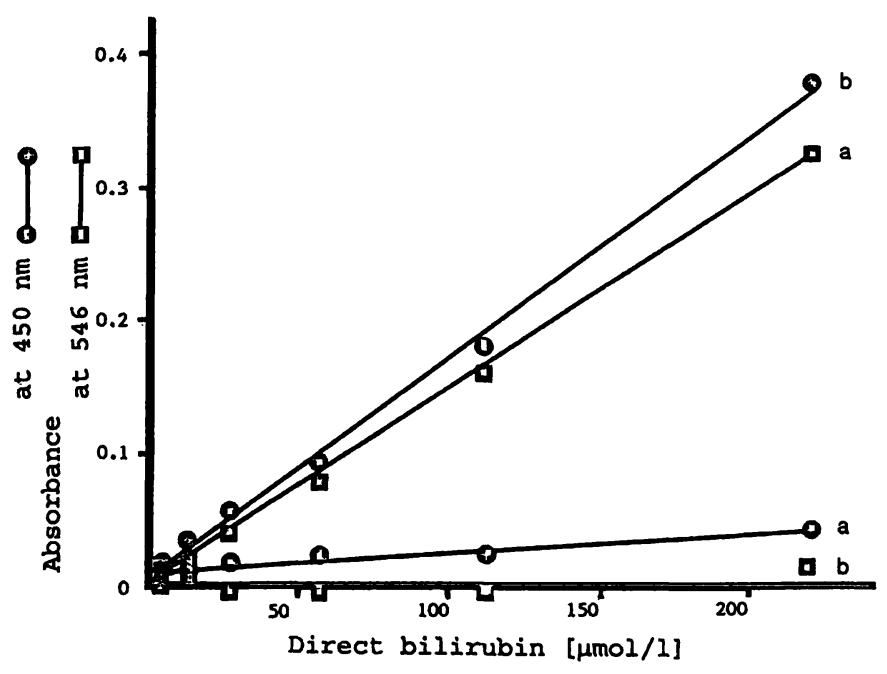

Fig. 4 Absorbance change before (b) and after (a) the reactions of the diazo $(\mathbf{0}-\mathrm{w})$ and the enzymatic $(0-0)$ methods. Values by the diazo method were measured at $546 \mathrm{~nm}$ while those by the enzymatic method at $450 \mathrm{~nm}$. The $x$-axis shows the direct bilirubin value of each sample measured by the diazo method. 


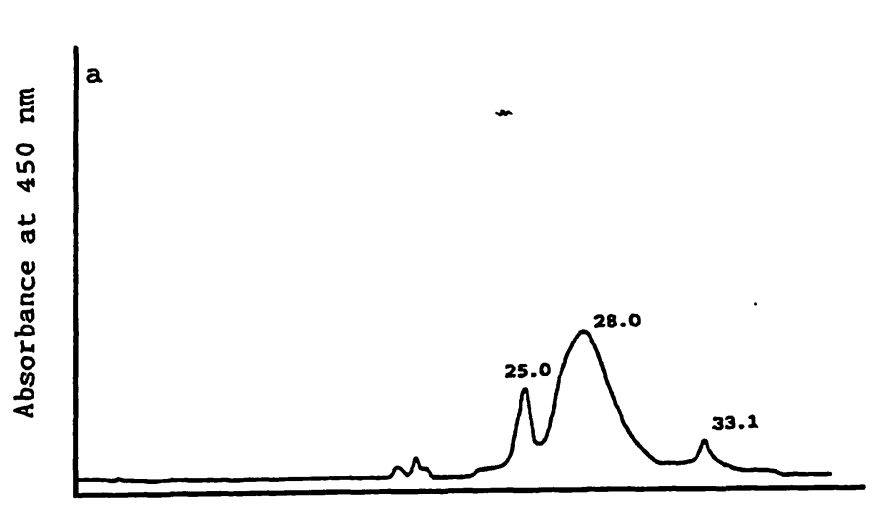

Retention time [min]

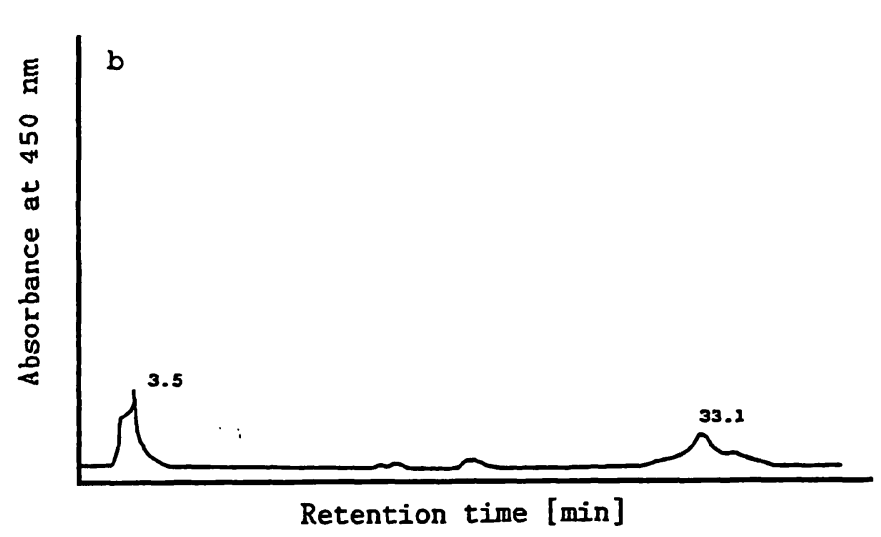

Fig. 5 Chromatogram of synthetic conjugated bilirubin before (a) and after (b) the addition of bilirubin oxidase at $\mathrm{pH} 3.7$ (for direct bilirubin measurement).

The number at each peak indicates the retention time ( $\mathrm{min})$.

min. At pH 7.2 (for total bilirubin measurement), this other material was not detected (fig. 6).

\section{Discussion}

It has been reported that measurement of $\delta$ bilirubin by the enzymatic method is not entirely accurate (9), and the same is true for the diazo method $(13,14)$. Thus, incomplete oxidation of $\delta$ bilirubin by bilirubin oxidase does not appear to be the main cause of the observed differences. In this analysis, type II hyperdirectbilirubinaemia ( $\alpha$ and $\beta$ were the main subfractions) showed little disparity (fig. 3 ), and unconjugated bilirubin ( $\alpha$ subfraction) also showed little disparity between the two methods (data not shown). It is therefore evident that $\delta$ bilirubin is not responsible for the observed discrepancies.

In the present study HPLC was used to reconfirm the complete oxidation of conjugated bilirubin by bilirubin oxidase (fig. 5) as reported by $L o$ et al. (13) and to reveal that the disparity depends on relative proportions of $\beta$ and $\gamma$ subfractions. On the other hand the disparity could be accounted for by the fact that the baseline of the enzymatic reaction rose according to the concentration of syn-
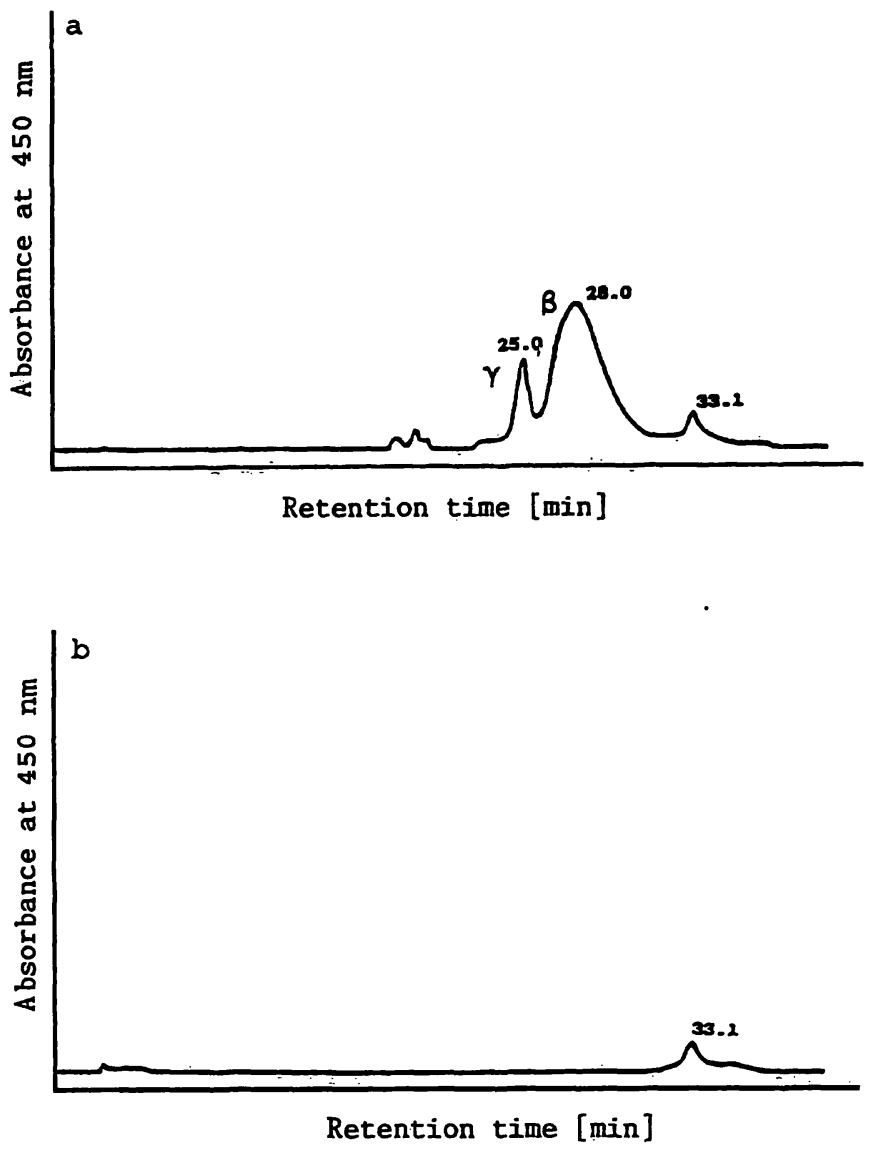

Fig. 6 Chromatogram of synthetic conjugated bilirubin before (a) and after (b) the addition of bilirubin oxidase at $\mathrm{pH} 7.2$ (for total bilirubin measurement).

The number at each peak indicates the retention time ( $\mathrm{min}$ ).

thetic conjugated bilirubin (ditaurobilirubin) (fig. 4). Also, HPLC analysis showed that the enzymatic reaction, when performed at $\mathrm{pH} 3.7$, yielded material absorbing at $450 \mathrm{~nm}$ which had a retention time of 2.9 to $3.5 \mathrm{~min}$ (fig. 6b), but not when performed at $\mathrm{pH} 7.2$ (complete oxidation conditions) (fig. 6b). It is suggested that this peak is due to incomplete oxidation products of ditaurobilirubin produced in the bilirubin oxidase reaction, since the absorbance increased in proportion to the ditaurobilirubin concentration and did not appear under the conditions of complete oxidation. Furthermore, the reactants include various substances absorbing at $450 \mathrm{~nm}$ on account of their broad peak width; but they do not include photobilirubin, one possible cause in the disparity, because this is attacked by bilirubin oxidase and its contribution to 450 $\mathrm{nm}$ absorbance is lost $(15,16)$.

Ditaurobilirubin is similar to conjugated bilirubin and serves as a suitable surrogate (17). Thus the main cause of the disparity is the incomplete oxidation of conjugated bilirubin by bilirubin oxidase. Bilirubin is completely oxidized if the $\mathrm{pH}$ in the reaction is raised. But the higher the $\mathrm{pH}$, the more unconjugated bilinubin that is oxidized. Raising the $\mathrm{pH}$ is therefore problematic, so that complete 
oxidation is difficult when measuring direct bilirubin. It is essential to take off this problem in the clinical interpreta- tion of bilirubin values measured by the enzymatic method.

\section{References}

1. Malloy HT, Evelyn KA. The determination of bilirubin with the photoelectric colorimeter. J Biol Chem 1937; 119:481-90.

2. Perry B, Doumas BT, Buffone G, Glick M, Ou CN, Ryder K. Measurement of total bilirubin by use of bilirubin oxidase. Clin Chem 1986; 32:329-32.

3. Doumas BT, Perry B, Jendrzejczak B, Davis L. Measurement of direct bilirubin by use of bilirubin oxidase. Clin Chem 1987; 33:1349-53.

4. Murao S, Tanaka N. A new enzyme "bilirubin oxidase" produced by Myrothecium verriucaria MT-1. Agric Biol Chem 1981; 45:2383-4.

5. Murao S, Tanaka N. Isolation and identification of a microorganism producing bilirubin oxidase. Agric Biol Chem 1982; 46:2031-4.

6. Jirsa M, Vecerek B, Ledvina M. Di- and mono-taurobilirubin similar to a directly reacting form of bilirubin in serum. Nature 1956; 177:895

7. Adachi $Y$, Inufusa $H$, Yamashita $M$, Kambe A, Yamazaki $K$, Sawada Y, et al. Clinical application of serum bilirubin fractionation by simplified liquid chromatography. Clin Chem 1987; 34:385-8

8. Ichida T, Nobuoka M. Ultramicro method for determination of total and direct bilirubin in serum by modified "alkaline azobilirubin blue" reaction. Clin Chem Acta 1968; 19:249-55.

9. Otsuji S, Mizuno K, Ito S, Kawahara S, Kai M. A new enzymatic approach for estimating total and direct bilirubin. Clin Biochem 1988; 21:38-8.

10. Kuenzle CC, Sommerhalder M, Ruttner JR, maier C. Separation and quantitative estimation of four bilirubin fractions from serum and of three bilirubin fractions from bile. J Lab Clin Med 1966; 67:282-93.

11. Wu TW, Lauff JJ, Kasper ME, Ambrose RT. Delta bilirubin: preliminary physico-chemical characterization and its implications in bilirubin determinations [abstract]. J Clin Chem Clin Biochem 1981; 19:881.

12. Wu TW. Delta bilirubin: the fourth fraction of bile pigments in human serum. Israel $\mathrm{J}$ Chem 1984; 23:241-7.

13. Lo $\mathrm{DH}, \mathrm{Wu} \mathrm{TW}$. Assessment of the fundamental accuracy of the Jendrassik-Gróf total and direct bilirubin assays. Clin Chem 1983; 29:31-6.

14. Lauff JJ, Kasper ME, Wu TW, Ambrose RT. Isolation and preliminary characterization of a fraction of bilirubin in serum that is firmly bound to protein. Clin Chem 1982; 28:629-37.

15. Ihara H, Aoki Y, Aoki T, Yoshida M. Light has a greater effect on direct bilirubin measured by the bilirubin oxidase method than by the diazo method. Clin Chem 1990; 36:895-7.

16. Ihara $H$, Nakamura $H$, Aoki $Y$, Aoki $T$, Yoshida $M$. In vitro effects of light on serum bilirubin subfractions measured by high-performance liquid chromatography: comparison with four routine methods. Clin Chem 1992; 38:2124-9.

17. Doumas BT, Wu TW, Poon KCP, Jendrzejczak B. Chemical nature of a synthetic bilirubin conjugate and its reactivities in the total and direct reactions by the Jendrassik-Grof method. Clin Chem 1985; 31:1677-82.

Dr. Katsushi Nakayama

Department of Pathology (I)

Juntendo University School of Medicine

2-1-1 Hongo Bunkyo-ku

Tokyo 113

Japan 


$$
\text { . }
$$

